\title{
Enfermedad celiaca e infertilidad no explicada: papel del tamizaje. Revisión de literatura.
}

\section{Celiac disease and unexplained infertility: role of screening. Literature review.}

Pág. 115,122

Recibido: 07-03-2020

Aceptado: $30-05-2020$

\author{
Dr. Ricardo Camacho Morales. Médico General, Costa Rica \\ Dr. Fabian Rodriguez Palma. Médico General. Caja Costarricense de Seguro Social, Costa Rica. \\ Dr. Camila Castro-Durán. Médico General, Costa Rica. \\ Dra. Mariana Villalobos Jímenez. Médico General, Costa Rica. \\ Dr. Yoel Merenstein Hoffman. Médico General, Costa Rica.
}

\section{Autor para correspondencia \\ Ricardo Camacho-Morales.}

\section{Conflictos de Interés}

Ninguno

\section{Financiamiento}

La presente investigación no ha recibido ayudas específicas provenientes de agencias del sector público, sector comercial o entidades sin ánimo de lucro.

\section{Resumen}

La enfermedad celíaca (EC) es un trastorno inflamatorio crónico causado por una respuesta inmune anormal inducida por una intolerancia permanente al trigo, la cebada y el centeno en la dieta. Dicho diagnostico se realiza por medio de pruebas sanguíneas de tamizaje en conjunto con confirmación endoscópica y biopsia. La EC se ha asociado a otras enfermedades autoinmunes y manifestaciones sistémicas. En los últimos años se ha estudiado con mayor relevancia la relación de EC con la infertilidad no explicada, dentro de los mecanismos fisiopatológicos se destaca la deficiencia nutricional y procesos autoinmunes especificos ya sea en el momento de la concepción o que estos mecanismos se desarrollen previo provocando deficiencia ovárica. Se ha propuesto realizar el tamizaje de EC en pacientes infertiles, sin embargo ha generado controversia debido a su utilidad. Si bien es cierto la relación entre dichas patologías es fuerte, se ha demostrado en los diferentes estudios que la utillidad en realizar dicho tamizaje de rutina depende del tipo de población y su prevalencia.

\section{Abstract}

Celiac disease $(C D)$ is a chronic inflammatory disorder caused by an abnormal immune response induced by a permanent intolerance to wheat, barley and rye in the diet. This diagnosis is performed by blood screening tests in conjunction with endoscopic confirmation and biopsy. CD has been associated with other autoimmune diseases and systemic manifestations. In recent years, the relationship of $C D$ with unexplained infertility has been studied with greater relevance. Among the pathophysiological mechanisms nutritional deficiency and specific autoimmune processes are highlighted either at the time of conception or that these mechanisms are priorly developed causing ovarian deficiency. CD screening in infertile patients has been proposed ;however, this has generated controversy due to its utility. Although the relationship between these pathologies is strong, it has been shown in different studies that the utility when performing such routine screening depends on the type of population and its prevalence. 
Palabras clave: Enfermedad celiaca, infertilidad, tamizaje.

Key words: Celiac disease, infertility, screening.

\section{Introducción}

La enfermedad celíaca (EC) es una enteropatía autoinmune causada por una respuesta inmune anormal a la ingesta de gluten, la fracción proteica encontrada en el trigo, cebada y centeno en pacientes genéticamente susceptibles conferida por las moléculas HLA clase II DQ2 o DQ8 (1). La prevalencia de la EC se reporta aproximadamente en $1 \%$, con una afectación principal en mujeres en una relación 3:1. Muchos investigadores han reportado asociaciones entre desórdenes extra-intestinales y celiaquía secundarios a las manifestaciones sistémicas de inflamación $(2,3)$.

La enfermedad también ha sido asociada a otras alteraciones autoinmunes como diabetes mellitus tipo 1, síndrome de Sjogren, tiroiditis de Hashimoto, entre otros (4). La forma clásica de esta patología se presenta durante la infancia como diarrea, anemia, distensión abdominal y retraso en el crecimiento. El inicio atípico en adultos ocurre en hasta un $45 \%$ de los casos y se presenta con dolor y distensión abdominal, osteoporosis y deficiencias nutricionales (2).

Algunos estudios sugieren una mayor prevalencia de EC no diagnosticada en pacientes infértiles, en un rango del $2-8 \%$ comparado con alrededor de un $1 \%$ en la población general, con ciertas variaciones en diferentes grupos étnicos; siendo mayor en europeos, regiones del oriente medio y África norte, y de forma inferior en países del sudeste de Asia $(5,6)$.

Se ha observado que la EC presenta una mayor prevalencia en mujeres, particularmente durante la edad reproductiva, pacientes en quienes se ha demostrado adicionalmente la presencia de múltiples desórdenes ginecológicos (6). Hasta el $15 \%$ de los casos de infertilidad son de causa desconocida y es en ellos en donde la búsqueda y el estudio de otras enfermedades de fondo tales como la EC toman más importancia; dado que un importante número de estas pacientes cursan asintomáticas y que la infertilidad puede ser en muchos casos la única manifestación (6).

La infertilidad en la EC no tratada puede deberse a los efectos sistémicos y endocrinológicos que tiene la malabsorción de hierro, folatos y déficits vitamínicos. Los primeros estudios respecto a la relación entre la EC y la infertilidad fueron realizados por Morris et al en 1970; donde se estudió un grupo de pacientes con EC no tratada e infertilidad no explicada, quienes consiguieron un embarazo exitoso luego del inicio de la dieta sin gluten; desde entonces su relación se ha estudiado más a profundidad (6).

\section{Diagnóstico}

De forma general, el diagnóstico de EC se inicia con pruebas sanguíneas de tamizaje con marcadores altamente sensibles y específicos: anticuerpo IgA contra endomisio (IgA-EMA) y anticuerpo IgA contra transglutaminasa tisular $(\lg A-T \operatorname{tg} \lg A)$. Las pruebas de tamizaje positivas requieren de confirmación con endoscopia y biopsia de intestino delgado. Una vez hecho el diagnóstico, el tratamiento es la adherencia de por vida a una dieta libre de gluten $(7,8)$.

Infertilidad ha sido definida como la falta de concepción posterior a un mínimo de 12 meses de relaciones sexuales sin protección (7). El abordaje de una paciente en estudio por infertilidad incluye pruebas de reserva ovárica (niveles de estradiol en día 2 y FSH y/o hormona Anti-Mulleriana $(\mathrm{AHM})$ ), histerosalpingografía, TSH, prolactina, y espermograma para la pareja masculina, y en algunos pacientes, laparoscopia como tamizaje por endometriosis (5). Se categoriza como infertilidad no explicada a aquellas pacientes que 
presentan resultados normales en un tamizaje básico por infertilidad (5).

Fisiopatología de los mecanismos de falla reproductiva inducidos por Enfermedad celíaca.

Los mecanismos patogénicos por los cuales se pueda explicar el papel de la EC en los desórdenes obstétricos asociados no son claros. Entre los que han sido propuestos destacan: deficiencia nutricional y procesos autoinmunes, de los cuales se pueden señalar los anticuerpos anti-TG de la circulación sanguínea materna que se unen a células trofoblásticas induciendo daño apoptótico, y también se ha descrito como a nivel materno, los anticuerpos anti-TG se unen a células endoteliales del endometrio causando una desarreglo del citoesqueleto de F-actina lo que afecta el proceso de angiogénesis $(1,9)$.

En cuanto a la hipótesis de malabsorción y deficiencia nutricional, que ocurre frecuentemente en EC activa, históricamente ha sido considerado como la principal causa de eventos adversos en el embarazo asociados a esta patología. El mecanismo propuesto para lo anteriormente mencionado consiste que a raíz de la estructura anormal de las vellosidades intestinales características de la celiaquía, resulta en un proceso de malabsorción que conlleva a anormalidades hematológicas menores, anemia y otras deficiencias nutricionales selectivas como zinc, selenio y ácido fólico (1).

La deficiencia de zinc y selenio ha mostrado provocar alteración de la síntesis y secreción de hormona luteinizante (LH) y Hormona foliculoestimulante (FSH), lo cual causa un eje hipófisis-ovárico anormal, amenorrea, abortos espontáneos y preeclampsia. La deficiencia de ácido fólico, por su papel como vitamina esencial en el metabolismo de ácidos nucleicos, tiene un impacto en los tejidos de rápida proliferación, como lo es el tejido embrionario, especialmente en su desarrollo neuronal (1).
Los estudios nutricionales en EC durante embarazo son muy limitados y la información es contradictoria. En algunos estudias se ha mostrado como la adherencia a una dieta libre de gluten significativamente aminora las alteraciones reproductivas en mujeres celíacas, mientras que por otro lado mujeres con infertilidad asociada a atrofia total o subtotal de las vellosidades usualmente no presentan malnutrición severa ni signos de deficiencia de elementos traza. Por lo anterior, el conocimiento actual no señala como mecanismo principal de las fallas reproductivas ocurridas en EC a las deficiencias nutricionales (1).

Dentro de los mecanismos autoinmunes propuestos, se puede destacar la apoptosis trofoblástica inducida por anticuerpos antiTG. Dichos anticuerpos han sido reportados como causa de daño neurológico e intestinal. La evidencia que apoya esta hipótesis es la expresión de TG en células endometriales, así como también en células placentarias estromales y trofoblásticas. La enzima TG está involucrada en el ensamblaje de matriz extracelular y adhesión celular, migración y crecimiento tisular, por ello se ha involucrado como parte de un rol crítico en el proceso de implantación. De esta forma, las TG en el sincitiotrofoblasto se unen a los anticuerpos anti-TG en pacientes con EC, lo cual podría afectar la evolución del embarazo (1).

Otra de las hipótesis planteadas como mecanismo autoinmune involucrado corresponde al efecto de los anticuerpos anti-TG en la angiogénesis endometrial. Se plantea que dichos anticuerpos al unirse con TG en la superficie celular del endotelio endometrial, interactúa con fibras de actina del citoesqueleto alterando su configuración y afectando su papel pivote en el proceso angiogénico. Lo cual se suma a que este mecanismo también se ha relacionado con la inhibición de los mediadores pro-angiogénicos

\section{(9) $(1) \Theta \theta$}


intracelulares ERK y FAK (1).

\section{Desórdenes ginecológicos}

Un número importante de diferentes factores pueden ser investigados en el caso de las pacientes con alteraciones en su fertilidad; dentro de los que se debe considerar la falla ovárica, la cual puede tener múltiples causas. La disfunción ovulatoria puede ocurrir en asociación con desórdenes endocrinológicos, particularmente los que se asocian con procesos mediados inmunológicamente como lo es la EC (10).

Dentro de las principales manifestaciones que se han observado en las pacientes celiacas se encuentra un retraso en la edad de aparición de la menarca, menopausia temprana, mayor incidencia de amenorrea, infertilidad y abortos espontáneos y en muchos casos recurrentes. En el caso de las pacientes infértiles se recomienda el tamizaje por EC luego de descartar anormalidades anatómicas, desórdenes endocrinos y enfermedades bacterianas del tracto urogenital, dado que posterior a que se aborden estas aristas y se descarten sus patologías, la infertilidad pasa a ser de etiología indefinida hasta demostrar lo contrario (6).

Hasta un $50 \%$ de pacientes con EC no tratada refieren haber tenido dificultades para concebir, la presencia de un aborto o un resultado desfavorable del embarazo; incluso se considera que en muchas pacientes con EC quienes cursan asintomáticas, el embarazo puede ser un desencadenante para que la enfermedad que había cursado de forma latente se vuelva clínicamente evidente (6).

Los desórdenes ginecológicos que suceden en estas pacientes se deben a múltiples etiologías. La deficiencia de zinc como antes se menciona es uno de los factores más correlacionados a explicar su fisiopatología (6).

\section{Tamizaje}

La decisión de llevar a cabo el tamizaje de EC en pacientes infértiles ha generado gran controversia y hasta el momento no se realiza de rutina. Sin embargo, debe tomarse en cuenta que la incidencia de dicha patología ha ido en aumento y que en pacientes con un diagnóstico de EC presentan un riesgo de hasta 8.9 veces mayor de presentar abortos espontáneos con respecto al resto de la población (6). Tomando en cuenta estas estadísticas, en la actualidad se debería realizar tamizaje por EC en aquellas pacientes infértiles una vez que se hayan descartado causas anatómicas, endocrinológicas y no se encuentre otra causa conocida que pueda explicar dicho trastorno.

Se ha demostrado que las pacientes celiacas que llevan una dieta sin restricciones, presentan un período reproductivo más corto con respecto al resto de la población. La edad de aparición de la menarca en niñas celiacas ha reportado retrasos de hasta 2 años y la aparición de la menopausia ha presentado adelantos de entre 2 y 3 años. En un estudio realizado por Molteni et al, se demostró que hasta un $38.8 \%$ de las pacientes no tratadas con EC de base refirieron amenorrea o al menos una alteración en sus ciclos menstruales; alteraciones que cedieron una vez que se iniciaron la dieta libre de gluten (6). Es importante diagnosticar la EC en la población con infertilidad inexplicada, dado que una simple intervención terapéutica como lo es la dieta sin gluten podría cambiar el desenlace gineco-obstétrico de estas pacientes (11).

\section{Discusión}

La EC es una patología presente en aproximadamente en $1-2 \%$ en la población general, como antes de menciona, por lo que se han realizado múltiples meta análisis de estudios 
de cohorte y estudios de casos y controles que han intentado dilucidar la asociación entres los desordenes reproductivos y la incidencia de EC. Estos estudios han documentado asociaciones entre infertilidad inexplicada, abortos recurrentes o restricción del crecimiento intrauterino, y el riesgo de EC $(1,12)$. Por lo contrario, aun no se existe literatura que evalué el impacto de una dieta libre de gluten en fertilidad o estudios prospectivos.

En el meta análisis realizado por Juneau et al, los autores describen la frecuencia con la que se han documentado pacientes con EC en poblaciones que han realizado fertilización in vitro (IVF). Además, se comparo las tasas de efectividad en mujeres con y sin anticuerpos positivos por EC; y finalmente compararon el éxito de la IVF $y$ del embarazo en pacientes que siguieron una dieta libre de gluten vs dieta normal. Los autores determinaron que la incidencia de EC en su población con IVF no era significativa en comparación a la población general. Por otra parte, se documento que la tasa de éxito de la IVF no vario significativamente entre paciente seropositivas o no, al igual que la mantener una dieta libre de gluten no afecto la tasa de éxito. Por lo tanto, se concluyó que realizar un tamizaje en esta población en estos momentos no cuenta con la evidencia que lo justifique en esta población (2). Los autores proponen que con los datos recolectados, surge la interrogante de si estos mismos resultados se mantendrían en una población con concepción natural que no requiera IVF, o si las mismas conclusiones se podrían aplicar a hombres infértiles con EC, o si solo aplican para parejas con diagnósticos de infertilidad específicos como infertilidad inexplicada. Se concluye que la dieta libre de gluten en la población infértil con $E C$, no muestra beneficio en las tasas de éxito de la IVF, se podría asumir que podrían tener mayor impacto en la calidad de vida en población con EC confirmada, donde esta dieta es tratamiento standard (2).
Otro estudio por Grode et al., demostró que realizar una prueba de tamizaje por EC en pacientes con IVF en un primer nivel representa una sensibilidad baja con una especificidad moderadamente buena. Esta prueba posee un alto valor predictivo negativo, sin embargo no se puede plantear como un reemplazo a los exámenes de laboratorio standard por la cantidad de falsos positivos. Este tamizaje se podría recomendar como prueba de triage para valorar la necesidad de completar con los exámenes de laboratorio pertinentes (13).

Un estudio por Kutteh et al., se basa en una población con antecedentes de perdidas recurrentes e infertilidad donde se valora la necesidad de tamizarlas por EC para determinar la existencia de una correlación entre perdidas gestacionales recurrentes y la presencia de seropositividad en marcadores de EC. Se ha demostrado en otros estudios, que existe una relación entre pacientes sintomáticas con EC activa y una alta tasa de perdidas gestacionales. Sin embargo el estudio actual indica que no existe un aumento en la prevalencia de marcadores para EC en una población asintomática, cabe resaltar que dicho estudio ha sido el que ha presentado una muestra más grande (14).

Dichos autores, indican que se documento que no existe diferencia significativa en la prevalencia de anticuerpos de EC al comparar poblaciones con abortos recurrentes con los controles. Como hallazgo incidental, se reporto que aproximadamente mas del $50 \%$ de la población con marcadores positivos por EC también poseían anticuerpo anticardiolipina (aPL) positivo. Se concluyo que, como se determino previamente en los estudios mencionados, no existe evidencia estadísticamente significativa para recomendar realizar tamizaje para EC en pacientes asintomáticas que curse con infertilidad. El estudio insta a realizar un diagnostico diferencial adecuado para indagar otras causas que expliquen las perdidas gestacionales, como

\section{(c) $(1) \Theta \Theta$}


la relación documentada con las pacientes con aPL positivo (14).

Otros estudios con poblaciones mas pequeñas sugieren que la presencia de los autoanticuerpos celiacos puede adversamente afectar el resultado obstétrico. Uno de estos reporta una relación estadísticamente significativa entre la EC y la amenaza de aborto, con un aumento de la posibilidad de presentar una complicación gestacional. Estos autores si recomiendan realizar screening de EC en pacientes muy sintomáticas con historia recurrente y persistente de desordenes reproductivos, donde se hayan descartado otras causas (15).

Estudios in vitro han documentado alteraciones fetales y complicaciones maternas como parte de su hipótesis en la falla obstétrica consecuencia de los autoanticuerpos de EC. Se sugiere que la exposición al gluten es estas pacientes genera respuestas autoinmunes que predisponen y conducen a complicaciones obstétricas como perdidas gestacionales recurrentes. Por lo que se sugiere que pacientes sintomáticas con EC e historia obstétrica con abortos recurrentes deberían ser estudiadas con biomarcadores como aPL y monitoreadas por complicaciones obstétricas (16).

\section{Conclusiones}

Se determinó que no se recomienda realizar tamizaje a pacientes asintomáticas por EC con historia de perdidas gestacionales recurrentes. Se comprobó que no existe una relación estadísticamente significativa en las pacientes con EC y estos antecedentes obstétricos. Además, se documentó que las medidas como implementar una dieta libre de gluten, tampoco cambiará el pronóstico o resultado del embarazo. Sin embargo estudios demuestran que realizar la prueba de tamizaje dependerá de la prevalencia de EC que exista en una población específica.

Por otra parte, según la literatura revisada, se propone que el tamizaje por EC se podría implementar en dos escenarios. El primero sería en pacientes con historia de EC que presenten antecedentes compatibles con infertilidad no explicada. En estos casos se podría realizar un tamizaje por autoanticuerpos por EC para valorar la necesidad de realizar otros estudios por anticuerpos o biomarcadores, por su elevado costo al ser realizados en primera instancia.

El otro escenario en el que la literatura recomienda realizar este tamizaje, es en pacientes con EC que se presenten sintomáticas con manifestaciones de perdidas gestacionales recurrentes. Esto por que se ha demostrado que pacientes con biomarcadores positivos por EC pueden presentar marcadores como aPL positivos hasta en $50 \%$, lo que podrían conllevar un aumento en el riesgo de presentar complicaciones obstétricas, por lo que

deberán ser monitoreadas durante su embarazo.

\section{Bibliografía}

1. Tersigni $C$, Castellani $R$, De waure $C$, Fattorossi A, De Spirito M, Gasbarrini A, et al. Celiac disease and reproductive disorders: Meta-analysis of epidemiologic associations and potential pathogenic mechanisms. Hum Reprod Update. 2014;20(4):582-93.

2. Juneau CR, Franasiak JM, Goodman LR, Marin D, Scott K, Morin SJ, et al. Celiac disease is not more prevalent in patients undergoing in vitro fertilization and does not affect reproductive outcomes with or without treatment: a large prospective cohort study. Fertil Steril [Internet]. 2018;110(3):437-42. Available from: https://doi. org/10.1016/j.fertnstert.2018.03.030

3. Nahar R, Aman A. Sealing the Diagnosis of Celiac Disease in Pregnancy. Med Forum. 2019;20(1). 
4. Rajput R, Chatterjee S. Primary infertility as a rare presentation of celiac disease. Fertil Steril [Internet]. 2010;94(7):2771.e5-2771. e7. Available from: http://dx.doi.org/10.1016/j. fertnstert.2010.04.032

5.Choi JM, Lebwohl B, Wang J, Lee SK, Murray JA, Sauer M V., et al. Increased prevalence of celiac disease in patients with unexplained infertility in the United states. J Reprod Med Obstet Gynecol. 2011;56(3):199-203.

6. Casella G, Orfanotti G, Giacomantonio L, Bella C Di, Crisafulli V, Villanacci V, et al. Celiac disease and obstetrical-gynecological contribution. Gastroenterol Hepatol from Bed to Bench. 2016;9(4):241-9.

7. Gunn B, Murphy KE, Greenblatt EM. Unexplained Infertility and Undiagnosed Celiac Disease: Study of a Multiethnic Canadian Population. J Obstet Gynaecol Canada [Internet]. 2018;40(3):2938. Available from: https://doi.org/10.1016/j. jogc.2017.07.008

8. Malhotra P, Malhotra N, Malhotra V, Gupta A, Chugh GA, Chaturvedi A, et al. Infertility and celiac disease. 2017;5:95-6.

9. Associate DMG, Senior M, Pgims PBDS. INTERNATIONAL JOURNAL OF SCIENTIFIC RESEARCH CELIAC DISEASE IN PATIENT WITH UNEXPLAINED INFERTILITY: A CASE Gastroenterology Sandeep Goyal * Manjri Virender Katyal. 2019;(12):8-9.

10. Freeman HJ. Infertility and ovarian failure in celiac disease. World J Obstet Gynecol. 2015;4(4):72.

11. Kumar A, Meena M, Begum N, Kumar N, Gupta RK, Aggarwal S, et al. Latent celiac disease in reproductive performance of women. Fertil Steril [Internet]. 2011;95(3):922-7. Available from: http:// dx.doi.org/10.1016/j.fertnstert.2010.11.005

12. Singh P, Arora S, Lal S, Strand TA, Makharia GK. Celiac Disease in Women with Infertility: A Meta-
Analysis. J Clin Gastroenterol. 2016;50(1):33-9. 13. Grode L, Møller Jensen T, Parkner T, Agerholm IE, Humaidan P, Hammer Bech B, et al. Diagnostic Accuracy of a Point-of-Care Test for Celiac Disease Antibody Screening among Infertile Patients. Inflamm Intest Dis. 2019;4(3):123-30.

14. Kutteh MA, Abiad M, Norman GL, Kutteh WH. Comparison of celiac disease markers in women with early recurrent pregnancy loss and normal controls. Am J Reprod Immunol. 2019;82(1):3-8.

15. Martinelli D, Fortunato F, Tafuri S, Germinario CA, Prato R. Reproductive life disorders in Italian celiac women. A case-control study. BMC Gastroenterol. 2010;10.

16. Di Simone N, De Spirito M, Di Nicuolo F, Tersigni C, Castellani R, Silano M, et al. Potential New Mechanisms of Placental Damage in Celiac Disease: Anti-Transglutaminase Antibodies Impair Human Endometrial Angiogenesis1. Biol Reprod. 2013;89(4):1-11. 


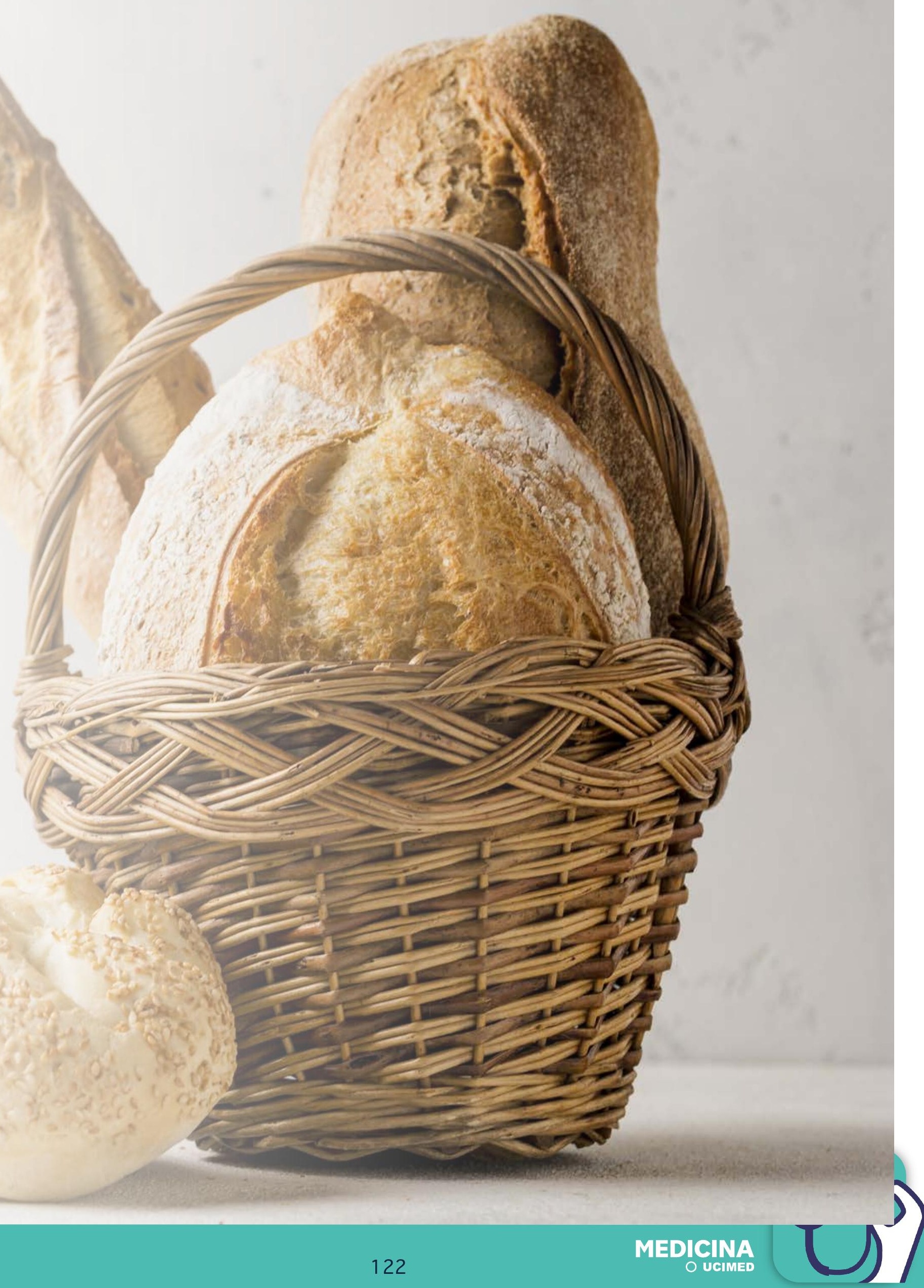

